\title{
Queueing theory-based path delay analysis of wireless sensor networks
}

\author{
Tie QIU ${ }^{1,2}$, Feng XIA ${ }^{1}$, Lin $\mathrm{FENG}^{2 *}$, Guowei WU ${ }^{1}$, Bo $\mathrm{JIN}^{2}$ \\ ${ }^{1}$ School of Software, Dalian University of Technology, Dalian 116620, China \\ ${ }^{2}$ School of Innovation Experiment, Dalian University of Technology, Dalian 116024, China \\ qiutie@dlut.edu.cn;f.xia@ieee.org;fenglin@dlut.edu.cn
}

\begin{abstract}
Path planning is one of the important factors that affect data transmission and processing in wireless sensor networks (WSNs). This paper addresses this issue by means of the paths delay analysis. Considering that WSNs are used for temperature monitoring, we model the WSNs using the open queueing network theory and analyze the paths delay based on the model. An iterative approximation algorithm is proposed for the qualitative analysis of the packet arrival rate of sensor nodes. According to the capacity and redundancy of nodes along the path, the destination node search trees are created for pre-selecting the transmission paths. Then the end-to-end delays of the pre-selected node paths and the average delay of sub-queueing networks are calculated. The optimal path and the assistant path for data transmission in WSNs could be obtained on the basis of the delay analysis. Numerical results demonstrate the effectiveness of the proposed approach.
\end{abstract}

Index Terms - wireless sensor networks, queueing networks, modeling, path planning, delay analysis

\section{INTRODUCTION}

Wireless sensor networks (WSNs) are a combination of many technologies, such as sensing, embedded computing, distributed information processing, and communications. The sensor nodes can collaborate on real-time monitoring, sensing, collecting network distribution of the various environments within the region or monitoring object information [1]. This information is then processed to obtain useful data, which will be sent to the user [2]. The power consumption of data transmission is maximal in WSNs [3], and the computing, storage, and communication capability of the node is more limited. Data are transmitted from the source node to target node. If data do not take a long time for searching the target node, the node in the network is always in the data transmission state. During this time, because the utilizations of nodes are not in equilibrium [4], the energy of individual nodes will run out because of the nodes' obvious failure $[5,6]$. This causes delay in transmission. Therefore, the problem of path planning, i.e., how to improve the speed of source node searching target node, is one of the research hot spots in the field of WSN.

A modeling method on large-scale network nodes is needed for optional paths in WSNs [7]. The current modeling method based on Petri nets [8] is suitable for macro-modeling, but it is not a modeling method that specifically suits large-scale WSNs. Queueing network is an

* Corresponding author

This work was supported in part by Natural Science Foundation of China under Grant No. 60773213 and No. 60903153, Program for New Century Excellent Talents in University (NCET-09-0251), the Fundamental Research Funds for the Central Universities (DUT10ZD110), and the SRF for ROCS, SEM. effective system-level modeling method, which is widely used in the modeling and performance analysis of computer systems and communication systems $[9,10]$. It has many advantages, which include a highly abstract and rich theory [11]. In recent years, research has made some progress about analysis and improving the network performance in the application of finite capacity queueing networks $[12,13]$. In [14] Bolot et al. gave a method of network path delay, in a relatively short time period detecting the shortest delay. In [15], Gurewitz et al. suggested an improved objective function based on the least-squares difference, and discussed the estimation method of deterministic time-delay. Papagiannaki et al. [16] measured the shortest delay of queueing system based on maximum entropy methods. Kouvatsos and Awan [17,18] described the priorities and blocking mechanisms with open-loop queueing network performance analysis, and queueing network parameters on the approximation and error estimates. Using queueing network delay evaluation, problems of analyzing modeling and path planning in various fields are successfully resolved. However, the topology of WSNs is very complex requiring a large number of transmission paths within the network. Thus, path delays are most computational overhead and slow down the speed. Therefore, performance evaluation using queueing network method for delay optimization is rarely mentioned in the field of WSNs. For example, Sheu et al. [19] compared and calculated end-to-end delays on different network sizes and a number of layers of IDGP protocol and DDGP protocol, but the number of network nodes was smaller, which did not require pre-selection paths. Bisnik et al. [20] modeled random access multi-hop wireless networks as open $\mathrm{G} / \mathrm{G} / 1$ queueing networks and used the diffusion approximation in order to evaluate closed form expressions for the average end-to-end delay, but it did not involve path planning.

This paper presents a path delay analysis method based on queueing network modeling of large-scale WSNs, which can be used e.g. in temperature monitoring. The approximate calculation method for packet arrival rate of node and the weighted value of the search tree algorithm are designed in order that the data transmission paths are pre-selected and the packet arrival rates are calculated. The end-to-end delays of node data transmission paths for pre-selecting and average delays of sub-queueing network in the entire system are compared to find an optimal data transmission path and alternate paths in the queueing networks for WSNs.

The remainder of this paper is organized as follows. Section II describes the problem to be solved and the definition of symbols used in the paper. In Section III, using 
an open queueing network model, the queueing network model for wireless sensor nodes is created according to data transfers, and the calculation method of end-to-end path delays and the system average delay are obtained in the open queueing network model. The approximate calculation method of packet arrival rate and weighted value of the search tree algorithm are designed in Section IV. Section V conducts a case study, in which the proposed method is applied to temperature monitoring using WSNs. The optimal path and alternate paths are obtained by paths analysis and numerical calculation. Section VI concludes the paper.

\section{Problem Statement}

Wireless sensor networks typically feature dynamic topology, limited energy, nodes with limited resources and non-reliability of data transmission. Therefore, they need real-time, economy, energy conservation and coordination in above four aspects to improve the network performance of WSNs and satisfy the performance requirements of the task scheduling system [21]. In practice, this should be done taking the relevant indicators into account. When the time of individual node communication is not normal or if there is energy depletion, the packets to be processed are queued in the blocking node. If the blocking can not be solved in time, blocking occurs in all the nodes of the link path, resulting in the congestion of the entire network. Then, a spare secondbest path except the optimal path becomes necessary to ensure that the entire WSN operation is efficient. This requires that to keeping the high efficiency of the path selection in a system for communication, and the searching paths occupy the smallest delay. Therefore, an effective modeling method of queueing network is explored necessarily to reduce the transmission delays of network at a minimal level when the system is blocked. At this time there should be a backup sub-optimal path (than the optimal path) to the work state to ensure a high efficiency operation in the WSNs.

As shown in Figure 1, WSNs are composed of sink nodes $[22,23]$, transmission nodes (i.e. nodes used to transmit packets), arrival nodes (through where packets arrive in the network) and leaving nodes (through where packets leave the network). There are three paths from node 11 to node 14 as shown in Figure 1(a). In the node path planning, it is easy to see that the path $\mathrm{P}(11 \rightarrow 12 \rightarrow 13 \rightarrow 14)$ is more redundant than $\mathrm{P}(11 \rightarrow 15 \rightarrow 14)$. Therefore, we have to consider the shortest delay path to transfer data in the design process of WSNs. However, it is not easy to make a concrete path by delay calculation between paths $\mathrm{P}(11 \rightarrow 15$ $\rightarrow 14)$ and $\mathrm{P}(11 \rightarrow 1 \rightarrow 14)$, because the packet arrival rate and processing speed of node 1 and node 15 are not the same. Note that packet arrival rate and processing speed are important to measure node performance. When the best path is determined, there should be provision for sub-optimal paths for the selection process to continue following the failure of the optimal path, to ensure the normal operation of the network. For large-scale WSNs, there are often multiple arrival nodes and multiple leaving nodes [24]. Figure 1(b) shows two sub-queueing networks: one from source node 11 to target node 14, and another from source node 16 to target node 17 . Figure $1(\mathrm{c})$ shows a WSN containing multiple clusters $[25,26]$, where transmission nodes 14 and 15 are the public nodes as the cluster 1 and cluster 2, and packets are that from source nodes 11 and 17 to target node 22 and 23 . The choice of the optimal transmission path becomes more complex as can be seen from Figure 1(a) to 1(c). We use queueing network modeling for path delays and sub-network delays will be calculated. Once end-to-end delays of node data transmission paths and average delays of the subqueueing networks are calculated, the data transmission path which is the optimal path and sub-optimal paths are determined.

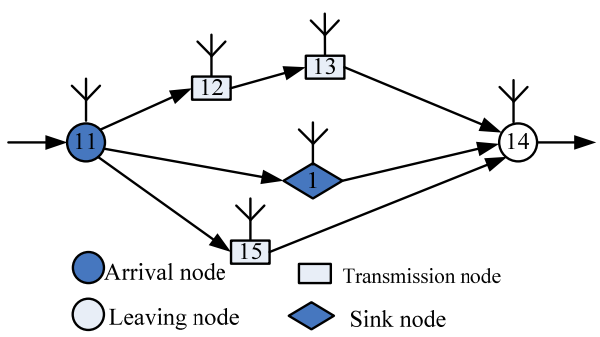

(a) Single arrival node to single leaving node

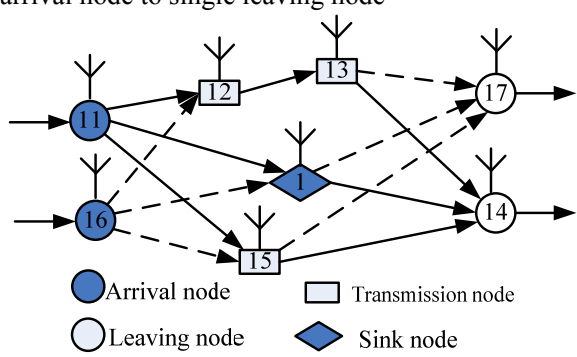

(b) Multiple arrival nodes to multiple leaving nodes

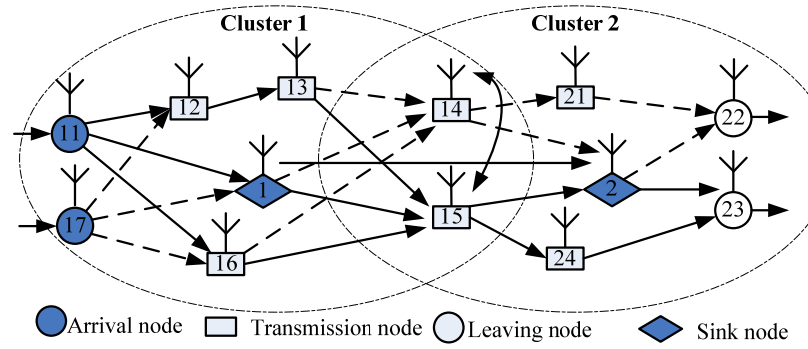

(c) A WSN with multiple clusters

Figure 1. Multi-path problem of WSNs

In order to facilitate the analysis of queueing network model for a WSN, the following symbols are defined as shown in Table I.

\section{THEORETICAL ANALYSIS}

\section{A. Open queueing network}

An open queueing network of a typical queue is shown in Figure 2. When a task reaches node $i$, there are three conditions in an open queueing network of a typical queue: (i) Independent external Poisson arrival $\lambda_{e}$. (ii) From queue $k$ with probability $p_{k i}$ to reach node $i$. (iii) With probability $p_{i i}$ in the network loop. When a task leaves from node $i$, there are two conditions: (i) To reach node $j$ with probability $p_{i j}$. (ii) To leave the node $i$ with probability $p_{i e}$. 
TABLE I. DEFINITION OF SYMBOLS

\begin{tabular}{|c|l|}
\hline Symbol & Description \\
\hline$i, j, k, d$ & Node number. \\
\hline$P(i \rightarrow j \rightarrow k)$ & Path from node $i$, through node $j$ to node $k$. \\
\hline$m$ & Number of paths to reach node $j$. \\
\hline$n$ & $\begin{array}{l}\text { Total number of nodes in the queueing } \\
\text { network. }\end{array}$ \\
\hline$N$ & Total number of nodes in the paths. \\
\hline$M$ & $\begin{array}{l}\text { Total number of paths in the queueing } \\
\text { network. }\end{array}$ \\
\hline$\lambda_{i}$ & Average task arrival rate of node $i$. \\
\hline$\mu_{i}$ & Service rate of node $i$. \\
\hline$\rho_{i}$ & Utilization of Node $i$. \\
\hline$\lambda_{e}$ & Independent external Poisson arrival. \\
\hline$p_{i e}$ & Probability of leaving the node $i$. \\
\hline$p_{i j}$ & Probability of from node $i$ to node $j$. \\
\hline$\gamma$ & $\begin{array}{l}\text { Pure packet arrival rate, which enter the } \\
\text { queueing network. }\end{array}$ \\
\hline$K$ & Node state. \\
\hline$E\left(k_{i}\right)$ & Average task number in the path $i$. \\
\hline$E(k)$ & $\begin{array}{l}\text { Average task number in the queueing } \\
\text { network. }\end{array}$ \\
\hline$E(T)$ & End-to-end delay with $N$-level nodes. \\
\hline$E_{M}(T)$ & Average network delay with $M$ paths. \\
\hline & \\
\hline &
\end{tabular}

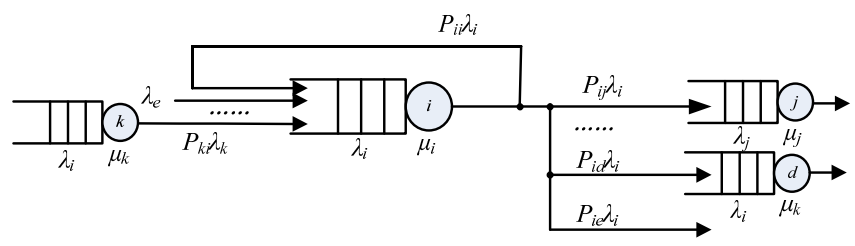

Figure 2. Queue modeling of a queueing network

Theorem 1. When an open queueing network enters a stable state, the sum of speeds of all tasks leaving a node equals that of all tasks entering the node.

$$
\begin{aligned}
& {\left[\lambda_{i}+\sum_{i=1}^{m} \mu_{i}\right] p(k)=\sum_{i=1}^{m} \lambda_{e} p\left(k-I_{i}\right)+\sum_{i=1}^{m} p_{i d} \lambda_{i} p\left(k+I_{i}\right)} \\
& +\sum_{i=1}^{m} \sum_{j=1}^{m} p_{k i} \lambda_{k} p\left(k+I_{j}-I_{i}\right)
\end{aligned}
$$

where $p(k)$ is the combinative probability in a stable state $k$, $I_{i}$ and $I_{j}$ are the unit vectors, which show the per unit change of a node starting from a state. More details on Theorem 1 can be found in [27].

\section{B. End-to-end path delay calculation}

Each queue delay is formed by queueing delay (wait delay) and transmission delay in the queueing network for WSNs. Transmission delay is linked with the arrival rate of packets and the service rate of nodes.

The utilization of node $i$ is given by:

$$
\rho_{i}=\frac{\lambda_{i}}{\mu_{i}}
$$

End-to-end delays caused by $\mathrm{M} / \mathrm{M} / 1$ queueing models of the $\mathrm{N}$-level nodes are explained in (3).

$$
E(T)=\sum_{i=1}^{N} \frac{1}{\mu_{i}-\lambda_{i}}=\sum_{i=1}^{N} \frac{1 / \mu_{i}}{1-\rho_{i}}
$$

\section{Average delay calculation for entire queueing network}

In the queueing network for wireless sensor networks, each node is considered as an $\mathrm{M} / \mathrm{M} / 1$ queue, and each path is considered as a queueing model $[27,28]$ with $N$-level serial nodes, service rate $\mu_{n}$, the average task arrival rate $\lambda_{n}$ and pure packet arrival rate $\gamma$ of entering the queueing network. The average number $E\left(k_{n}\right)$ of tasks in the path $m$ equals the sum of servicing and queueing packets. $E\left(k_{n}\right)$ can be obtained through (4).

$$
E\left(k_{n}\right)=\lambda_{n} T_{n}=\frac{\lambda_{n}}{\mu_{n}-\lambda_{n}}
$$

The average number of packets $E(k)$ in a queueing network path can be obtained from (5).

$$
E(k)=\sum_{n=1}^{m} E\left(k_{n}\right)
$$

The relationship between the average delay $E_{M}(T)$ and the average number $E(k)$ of tasks in a queueing network can be described as follows.

$$
\gamma E(T)=E(k)
$$

The average network delay with $M$ paths can be obtained from (4), (5), and (6), which is shown in (7).

$$
E_{M}(T)=\frac{1}{\gamma} \sum_{n=1}^{M} \lambda_{n} T_{n}=\frac{1}{\gamma} \sum_{n=1}^{M} \frac{\lambda_{n}}{\mu_{n}-\lambda_{n}}
$$

According to (7), we can calculate the average network delay of the sub-queueing network in WSNs.

\section{Algorithm Design}

\section{A. Approximate iterative algorithm for packet arrival rates}

The data transfer between each node is mutual in the queueing network for wireless sensor networks. Therefore, the calculation for the packet arrival rate of a previous node may be used as the unknown packet arrival rate of earlier computing nodes. In a lot of practical application and engineering experiments, we found that when a node communication of WSNs enters into a stable state, the average packet arrival rate of node tends to be a constant value. We designed an iterative method, which shown in Algorithm 1. We set initial values to the network status, and then gradually revise the last time packet arrival rate by our iterative method. In the end, a system of approaching the equilibrium was found.

\begin{tabular}{l}
\hline Algorithm 1 \\
\hline Begin \\
connection for each node was obtained in the queueing \\
network model. External packet arrival rates $\lambda_{j}^{e}(j$ is a \\
node number of arrival node) are determined. \\
Step 2. Initialize total packet arrival rates $\lambda_{i}^{0}$ ( $i$ is a node \\
number in the network) of the $n$ nodes. \\
Step 3. For queue $j$ in queueing network, the total packet \\
arrival rate can be calculated as follows: \\
$\qquad \lambda_{j}=\lambda_{j}{ }^{e}+\sum_{i=1}^{m} \lambda_{i} p_{i j}$ \\
According to the connection of each node in the \\
queueing network model of Step 1 , create the following \\
constraints: \\
$\qquad \lambda_{j}^{1}=\lambda_{j}^{e}+\sum_{i=1}^{m} \lambda_{i}^{0} p_{i j}$
\end{tabular}


Using the results of (9), the next node of the packet arrival rate is calculated as Equation (10) shows

$$
\lambda_{j+1}^{1}=\lambda_{j+1}^{e}+\sum_{i=1}^{j} \lambda_{i}^{1} p_{i j}+\sum_{i=j+1}^{m} \lambda_{i}^{0} p_{i j}
$$

Thus the total packet arrival rates of nodes are calculated.

Step 4. We use $\lambda_{j}^{1}$ to amend the internal packet arrival rates for every node, where $j$ denotes node number.

Step 5. If the difference between the internal packet arrival rates for two computing tasks (before and after) is less than a certain value, then go to Step 6. Otherwise jump to Step 3 followed by iterative calculation.

Step 6. Return the total packet arrival rate of each node $\lambda_{j}^{\theta}$ (where $\theta$ is the number of iterations).

End

\section{B. Path pre-selection algorithm}

The number of nodes required for a WSN that consists of an open queueing network is very large. If we need to calculate the time delay of each node and then determine the optimal path, computation will be extensive.

\begin{tabular}{|l|}
\hline Algorithm 2 \\
\hline Begin \\
Step 1. The connection of each node is obtained \\
according to the transition probability of a queueing \\
network model, as well as that of the network reaching \\
and transferring figure.
\end{tabular}

Step 2. Packets leaving from the node $i$ with external arrival rate $\lambda_{i}^{e}$ to node $j$ through the queueing network.

On the basis of the following rules start building the search tree from node $i$.

Step 2.1. Node $i$ is the root node.

Step 2.2. According to node transition relationship, add the leaf node.

Step 2.3. For sub-tree generated by non-sink node, if two node numbers, which leaf node and its parent node and sibling node of parent node are same, this leaf node is invalid.

Step 2.4. The leaf node is deleted. Before searching the destination node $j$, if the non-sink node is not added, go back to Step 2.2; otherwise, go to Step 2.5.

Step 2.5. When sink node $k$ is met, node $k$ becomes the root of the sub-tree, go to Step 2.2 until non-sink nodes are completed to add.

Step 2.6. The sub-tree generated by sink node $k$ is inserted into the main tree of the root as node $i$.

Step 3. The resulting search tree is completely traversed from the root node $i$. Thus, all the paths to reach node $j$ are obtained.

Step 4. According to the node service rate as the weight, comparison of the output paths is to remove the path with redundant nodes.

Step 5. The pre-selection paths from node $i$ to node $j$ in a queueing network take to be output.

End

Especially for a large queueing network model, clusters and other high performance processors may be used to complete the computing task. Therefore, we pre-selected the paths between source node and destination node before calculating the path delay, as shown in Algorithm 2.

\section{NuMERICAL RESUltS}

\section{A. Task analysis and modeling}

We conduct a case study of WSNs for monitoring temperature. The network topology is shown in Figure 3. The wireless sensor network is divided into two clusters.

In cluster 1 , node 11 and node 12 are arrival nodes, nodes $13,14,15$ and 16 are transmission nodes, and node 1 is the sink node which is responsible for communications with the inner cluster nodes and adjacent cluster node 2 . In cluster 2 , node 21 and node 22 are transmission nodes, nodes 23, 24, 25 and 26 are leaving nodes, and node 2 is the sink node which is responsible for communications with the inner sensor nodes and adjacent sink node 1 .

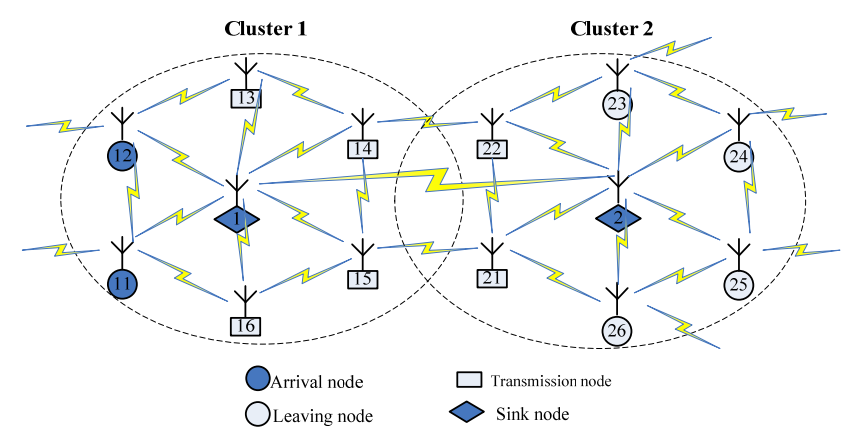

Figure 3. Topology of a wireless sensor network

The transfer of data packets $r$ between each node for temperature monitoring is analyzed, and data transition probabilities between each node are obtained. According to the network structure, topology and data transfers can be obtained through an open queueing network model with the transition probability, as shown in Figure 4. In the figure, the connection line with arrow means the direction of data flow, and the numbers on the line indicate transition probability.

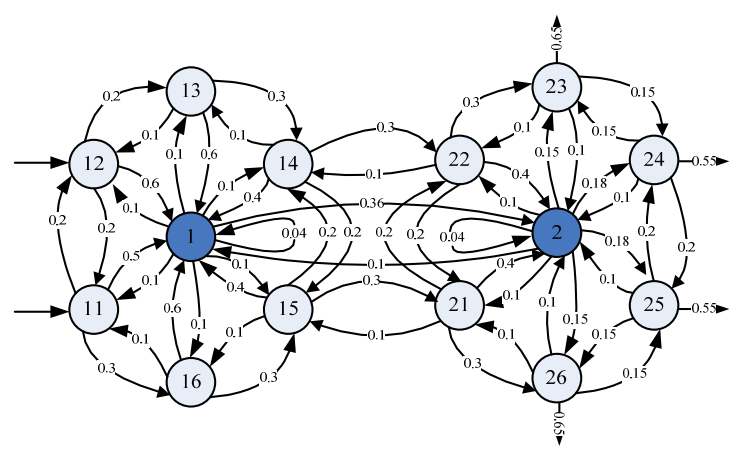

Figure 4. Queueing network model of the WSN

\section{B. End-to-end delay calculation and path analysis}

The external packet arrival rate of node 12 is 15 packets per second, and the external packet arrival rate of node 13 is 10 packets per second. According to algorithm 1, convergent $\lambda_{11}, \lambda_{12}, \ldots, \lambda_{16}, \lambda_{1}, \lambda_{21}, \lambda_{22}, \ldots, \lambda_{26}$, and $\lambda_{2}$ are obtained. The packet arrival rate transfer matrix (11) is obtained as follows. 


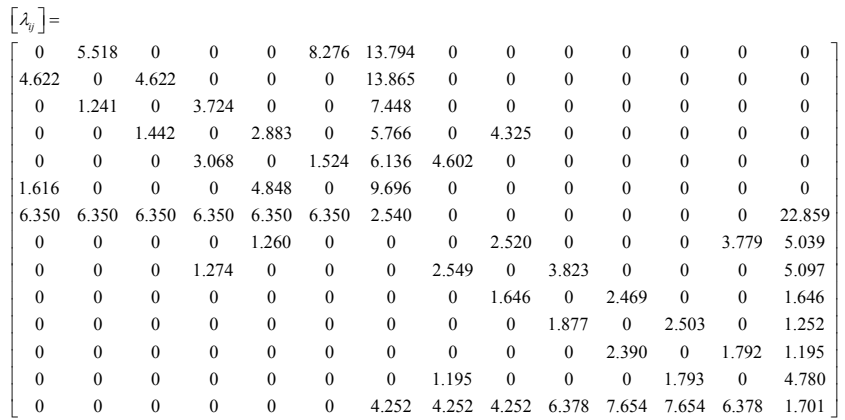

$(11)$

Nodes of different types have different service rates. The service rate of arrival nodes and leaving nodes is 20 packets per second, and the service rate of transmission nodes is 30 packets per second. The service rate of sink nodes is 60 packets per second. With this information, we can calculate any queueing path delay in the wireless sensor network. For the large number of queues in the network path, if the delay of each path is to be calculated, the computing task is very huge. We need first to pre-select the path using Algorithm 2. The paths starting from node 11 are listed as follows. The delay analysis for paths starting from node 12 is omitted here due to space limit.

From node 11 to node 23:

$$
\begin{aligned}
& \mathrm{P} 1:(11 \rightarrow 1 \rightarrow 2 \rightarrow 23) \\
& \mathrm{P} 2:(11 \rightarrow 1 \rightarrow 14 \rightarrow 22 \rightarrow 23) \\
& \mathrm{P} 3:(11 \rightarrow 1 \rightarrow 15 \rightarrow 21 \rightarrow 26 \rightarrow 25 \rightarrow 24 \rightarrow 23) \\
& \mathrm{P} 4:(11 \rightarrow 12 \rightarrow 13 \rightarrow 14 \rightarrow 22 \rightarrow 23) \\
& \mathrm{P} 5:(11 \rightarrow 16 \rightarrow 15 \rightarrow 14 \rightarrow 22 \rightarrow 23) \\
& \mathrm{P} 6:(11 \rightarrow 16 \rightarrow 15 \rightarrow 21 \rightarrow 22 \rightarrow 23) \\
& \mathrm{P} 7:(11 \rightarrow 16 \rightarrow 15 \rightarrow 21 \rightarrow 2 \rightarrow 23) \\
& \mathrm{P} 8:(11 \rightarrow 16 \rightarrow 15 \rightarrow 21 \rightarrow 26 \rightarrow 25 \rightarrow 24 \rightarrow 23)
\end{aligned}
$$

From node 11 to node 24:

$$
\begin{aligned}
& \mathrm{P} 1:(11 \rightarrow 1 \rightarrow 2 \rightarrow 24) \\
& \mathrm{P} 2:(11 \rightarrow 1 \rightarrow 15 \rightarrow 21 \rightarrow 26 \rightarrow 25 \rightarrow 24) \\
& \mathrm{P} 3:(11 \rightarrow 1 \rightarrow 14 \rightarrow 22 \rightarrow 23 \rightarrow 24) \\
& \mathrm{P} 4:(11 \rightarrow 16 \rightarrow 15 \rightarrow 21 \rightarrow 22 \rightarrow 23 \rightarrow 24) \\
& \mathrm{P} 5:(11 \rightarrow 16 \rightarrow 15 \rightarrow 21 \rightarrow 26 \rightarrow 25 \rightarrow 24) \\
& \mathrm{P} 6:(11 \rightarrow 16 \rightarrow 15 \rightarrow 21 \rightarrow 2 \rightarrow 24) \\
& \mathrm{P} 7:(11 \rightarrow 12 \rightarrow 13 \rightarrow 14 \rightarrow 22 \rightarrow 23 \rightarrow 24)
\end{aligned}
$$

From node 11 to node 25:

$$
\begin{aligned}
& \mathrm{P} 1:(11 \rightarrow 1 \rightarrow 2 \rightarrow 25) \\
& \mathrm{P} 2:(11 \rightarrow 1 \rightarrow 15 \rightarrow 21 \rightarrow 26 \rightarrow 25) \\
& \mathrm{P} 3:(11 \rightarrow 16 \rightarrow 15 \rightarrow 21 \rightarrow 2 \rightarrow 25) \\
& \mathrm{P} 4:(11 \rightarrow 16 \rightarrow 15 \rightarrow 21 \rightarrow 26 \rightarrow 25)
\end{aligned}
$$

From node 11 to node 26:

$$
\begin{aligned}
& \mathrm{P} 1:(11 \rightarrow 1 \rightarrow 2 \rightarrow 26) \\
& \mathrm{P} 2:(11 \rightarrow 1 \rightarrow 15 \rightarrow 21 \rightarrow 26) \\
& \text { P3:(11 } \rightarrow 1 \rightarrow 14 \rightarrow 22 \rightarrow 21 \rightarrow 26) \\
& \text { P4:(11 } \rightarrow 16 \rightarrow 15 \rightarrow 21 \rightarrow 26)
\end{aligned}
$$

The delays of the above paths are compared in Figure 5.

As can be seen from Figure 5, the path P1 leaving from node 23 occupies the shortest delay, and P2 comes second. If the communication between sink node 1 and sink node 2 is blocked, we can consider using path $\mathrm{P} 2$ as the transmission path. The path P1 leaving from node 24 occupies the shortest delay, and other paths P3 and P6 have similar delays. In practical applications, we can make path $\mathrm{P} 1$ as a high priority path, and paths $\mathrm{P} 3$ and $\mathrm{P} 6$ as auxiliary transmission paths. The path P1 leaving from node 25 occupies the shortest delay, which can be taken as the preferred path to transfer data. Among other paths, paths P2 and P3 are relatively shorter. When the communications between sink nodes 1 and 2 are blocked, we can consider using the path $\mathrm{P} 2$ or P3 as the transmission path. Path P1 leaving from node 26 occupies the shortest delay. P2 is the second transmission path for standby. When the communication between sink nodes 1 and 2 is blocked, we can consider using path $\mathrm{P} 2$ as the transmission path.

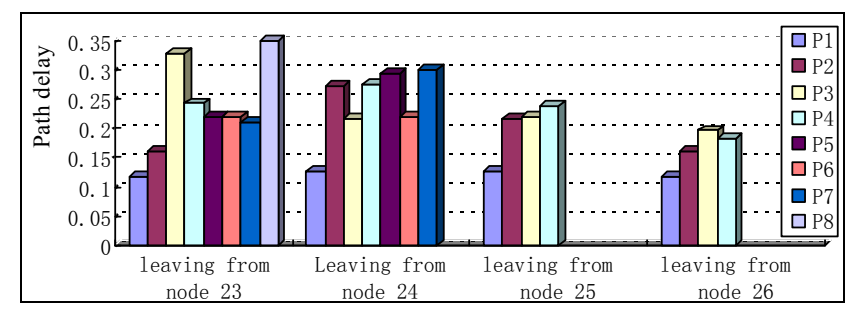

Figure 5. Delays of paths starting from node 11

It is clear from the above analysis that the communication between the sink nodes is the main mode of information transfer between the clusters and other preferential nodes as a secondary priority communications.

\section{Calculation of average delay}

The average delay of a queueing network is an important indicator to measure the integrated processing of all nodes in the system. The 8 sub-queueing networks are composed of paths starting separately from node 11 and node 12 to reach nodes 23, 24, 25 and 26, then leaving the path. Using the method for calculating the average delay of a queueing network in subsection $\operatorname{III}(\mathrm{C})$, the sub-queueing networks with the various $M$ paths are calculated. The delay comparison is shown in Figure 6.

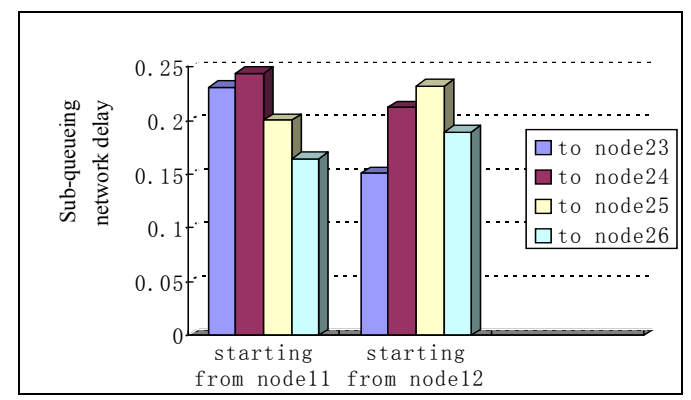

Figure 6. Average network delay comparison of each sub-queueing networks

As can be seen from Figure 6, the path of starting from node 11 to reach node 26 occupied the shortest delay. The path starting from node 12 to reach node 23 occupied the shortest delay. Therefore, the path starting from node 11 to reach node 26, and then leaving the path can be the preferred path to transfer data. In other cases, the path starting from node 12 to reach node 23 , and then leaving the path can be the preferred path to transfer data in traffic routing of wireless sensor network for monitoring temperature. 


\section{CONCLUSIONS}

This paper has presented a method of modeling and delay analysis for WSNs based on open queueing network. Based on the principles of data transmission of WSNs, an open queueing network model is established. Using that model, an approximate iterative algorithm is designed to calculate node packet arrival rate, when the system communication is stable. As the dimensions of WSNs are very large, the calculation is very difficult if the end-to-end delay of each path in the queueing network is to be calculated. In order to speed up the calculation, we have designed the pre-selection algorithm based on a path search tree. The redundant paths are removed according to node service rate as weight value. Thus, the number of pre-selecting paths is much smaller than the number of actual paths in the wireless sensor network. Using the analysis method of end-to-end delay for pre-selecting paths, the optimal path and sub-optimal paths are obtained. Finally, the average delay of sub-queueing networks which are composed of pre-selecting paths is calculated, and the sub-queueing network with optimal average queueing delay is obtained. This work provides an effective solution, which could help in the selection of a data transmission path for large-scale WSNs, and increase the data transmission efficiency of WSNs.

The queueing network model of a single-server M/M/1type system for WSNs is discussed in this paper. A multiserver situation (i.e., a node with multiple parallel processors, for example, the sink node in a multiple-server $\mathrm{M} / \mathrm{M} / \mathrm{m}$-type system) will be explored in future work, as well as dealing with the blocking between nodes.

\section{REFERENCES}

[1] E. Onur, C. Ersoy, H. Deliç, and L. Akarun, "Surveillance with wireless sensor networks in obstruction: Breach paths as watershed contours," Computer Networks, vol. 54, no. 3, pp. 428-441, Feb 2010, Available: http://dx.doi.org/10.1016/j.comnet.2009.09.006.

[2] J. Li, and H. Gao, "Survey on sensor network research," Journal of Computer Research and Development, vol. 45, no. 1, pp. 1-15, Feb. 2008.

[3] H. Jeon, J. Choi, H. Lee, and J. Ha, "Channel-aware energy efficien transmission strategies for large wireless sensor networks," IEEE Signal Processing Letters, vol. 17, no. 7, pp. 643-646, Dec. 2010 Available: http://dx.doi.org/10.1109/GLOCOM.2010.5683415.

[4] P. S. Sausen, M. A. Spohn, and A. Perkusich, "Broadcast routing in wireless sensor networks with dynamic power management and multicoverage backbones," Information Sciences, vol. 180, no. 5, pp. $653-$ 663, Mar. 2010, Available: http://dx.doi.org/10.1016/j.ins.2009.11.016

[5] Y. Tang, M. Zhou, and X. Zhang, "Overview of routing protocols in wireless sensor networks," Journal of Software, vol. 17, no. 3, pp 410-421, Mar. 2006

[6] H. Chen, C. K. Tse, and J. Feng, "Impact of topology on performance and energy efficiency in wireless sensor networks for source extraction," IEEE Transactions on Parallel and Distributed Systems, vol. 20, no. 6, pp. 886-897, 2009, Available: http://dx.doi.org/10.1109/TPDS.2009.14.

[7] G. Li, C. Zhu, and X. Li, "Application of chaos theory and wavelet to modeling the traffic of wireless sensor networks," 2010 International Conference on Biomedical Engineering and Computer Science, ICBECS, pp. 1-4, Wuhan, Apr. 2010: Available: http://dx.doi.org/10.1109/ICBECS.2010.5462503.

[8] A. Shareef, and Y. Zhu, "Energy modeling of processors in wireless sensor networks based on petri nets," Proceedings of the International Conference on Parallel Processing Workshops, pp. 129-134, Portland, Sep. 2008, Available: http://dx.doi.org/10.1109/ICPP-W.2008.35.
[9] B. Vidhyacharan, "State diagrams and steady-state balance equations for open queueing network models," Computers and Electrical Engineering,vol. 31, no. 7, pp. 460-467, Oct. 2005, Available: http://dx.doi.org/10.1016/j.compeleceng.2005.09.001.

[10] J. N. Daigle, Queueing theory with applications to packet telecommunication, Boston, MA: Springer Science and Business Media, Inc., 2005.

[11] L. Wang, Z. Wang, and K. Dai, “An approximate method by queueing network modeling for performance evaluation of asynchronous pipeline rings," 2006 IEEE Int Conf on Computer and Information Technology, pp. 244-249, Seoul, sep. 2006, Available: http://dx.doi.org/10.1109/CIT.2006.38.

[12] A. A. A. Kock, L. F. P. Etman, and J. E. Rooda, "Effective process times for multi-server flowlines with finite buffers," IIE Transactions, vol. 40, no. 3, pp. 177-186, Mar. 2008, Available: http://dx.doi.org/10.1080/07408170701488029.

[13] M. V. Vuuren, I. J. B. F. Adan, and S. A. E. Resingsassen, "Performance analysis of multi-server tandem queues with finite buffers and blocking," OR Spectrum, vol. 27, no. 2-3, pp. 315-338, 2005, Available: http://dx.doi.org/10.1007/s00291-004-0189-z

[14] J. C. Bolot, "Characterizing end-to-end packet delay and loss in the internet," Journal of High Speed Networks, vol. 2, no. 3, pp. 305, 1993.

[15] O. Gurewitz, I. Cidon, and M. Sidi, "One-way delay estimation using network-wide measurements," IEEE Transactions on Information Theory, vol. 52, no. 6, pp. 2710-2724, Jun. 2006, Available: http://dx.doi.org/10.1109/TIT.2006.874414.

[16] K. Papagiannaki, S. Moon, C. Fraleigh, and et al., "Analysis of measured single-hop delay from an operational backbone network," Proceedings of IEEE INFOCOM'02. pp. 535-544, New York, IEEE, Jun. 2002, Available: http://dx.doi.org/10.1109/INFCOM.2002.1019298.

[17] D. Kouvatsos, and I. Awan, "Entropy maximisation and open queueing networks with priorities and blocking," Performance Evaluation, vol. 51, no. 2-4, pp. 191-227, Feb. 2003, Available: http://dx.doi.org/10.1016/S0166-5316(02)00092-5.

[18] I. Awan, "Analysis of multiple-threshold queues for congestion control of heterogeneous traffic streams," Simulation Modelling Practice and Theory, vol. 14, no. 6, pp. 712-724, Aug. 2006, Available: http://dx.doi.org/10.1016/j.simpat.2005.10.004.

[19] J. Sheu, P. K. Sahoo, C. Su, and W. Hu, "Efficient path planning and data gathering protocols for the wireless sensor network," Computer Communications, vol. 33, no. 3, pp. 398-408, Feb. 2010, Available: http://dx.doi.org/10.1016/j.comcom.2009.10.011.

[20] N. Bisnik, and A. A. Abouzeid, "Queueing network Models for Delay Analysis of Multihop Wireless Ad Hoc Networks," Ad Hoc Networks, , vol. 7, no. 1, pp. 79-97, Jan. 2009, Available: http://dx.doi.org/10.1016/j.adhoc.2007.12.001.

[21] G. Chen, W. Guo, and Y. Chen, "Research on dynamic alliance of task allocation and its algorithm in wireless sensor network," Journal on Communications, vol. 30, no. 11, pp. 48-55, 2009.

[22] J. Yick, B. Mukherjee, and D. Ghosal, "Wireless sensor network survey," Computer Networks, vol. 52, no. 12, pp. 2292-2330, Aug 2008, Available: http://dx.doi.org/10.1016/j.comnet.2008.04.002.

[23] I. F. Akyildiz, W. Su, Y. Sankarasubramaniam, and E. Cayirci, “A survey on sensor networks," IEEE Communications Magazine, vol. 40, no. 8, pp. 102-114, Aug. 2002, Available: http://dx.doi.org/10.1109/MCOM.2002.1024422.

[24] J. Son, J. Lee, and S. Seo, "Topological key hierarchy for energyefficient group key management in wireless sensor networks," Wireless Personal Communications, vol. 52, no. 2, pp. 359-382 Jun. 2010, Available: http://dx.doi.org/10.1007/s11277-008-9653-4

[25] S. A. B. Awwad, C. K. Ng, N. K. Noordin, and M. F. A. Rasid, "Cluster Based Routing Protocol for Mobile Nodes in Wireless Sensor Network," Wireless Personal Communications, vol. 55, no. 5 , pp. 1-31, 2010, Available: http://dx.doi.org/10.1007/10.1007/s11277010-0022-8.

[26] H. Abusaimeh, and S. Yang. Dynamic cluster head for lifetime efficiency in WSN. International Journal of Automation and Computing, vol. 6, no. 1, pp. 48-54, Feb. 2009, Available: http://dx.doi.org/10.1007/s11633-009-0048-0.

[27] Y. Sheng. Queueing Theory and Its Application in Modern Communication. BeiJing, Posts \& Telecom Press, 2007.

[28] T. Qiu, L. Wang, H. Guo, L. Feng, and L. Shu, "A new modeling method for vector processor pipeline using queueing network," 5 th International ICST Conference on Communications and Networking, pp. 1-6, Beijing, Aug. 2010. 